\title{
AN APPROACH FOR DETECTION OF GLOMERULI IN MULTISITE DIGITAL PATHOLOGY
}

\author{
R. Marée ${ }^{a, b}$, S. Dallongeville ${ }^{a}$, J.-C. Olivo-Marin ${ }^{a}$, V. Meas-Yedid ${ }^{a}$ * \\ ${ }^{a}$ BioImage Analysis Unit, CNRS UMR 3691, \\ Institut Pasteur, 25 rue du docteur Roux, 75015, Paris, France \\ ${ }^{\mathrm{b}}$ University of Liège, Systems and Modeling \\ Montefiore Institute \& GIGA-Research, Grande Traverse 10, 4000 Liège Sart-Tilman, Belgique
}

\begin{abstract}
We present a novel bioimage informatics workflow that combines Icy and Cytomine software and their algorithms to enable large-scale analysis of digital slides from multiple sites. In particular, we apply this workflow on renal biopsies and evaluate empirically our approach for the automatic detection of glomeruli in hundreds of tissue sections.
\end{abstract}

Index Terms - whole slide imaging, image processing, color normalization, machine learning, glomeruli detection

\section{INTRODUCTION}

Digital pathology is an active field of research which raises many image analysis challenges [14]. One of the key challenge for pattern recognition algorithm is the wide variety of preparation and imaging protocols that imply highly variable image appearances of tissue structures, within a given laboratory and across research centers. Indeed, tissue sections are prepared using coloured histochemical stains that bind selectively to cellular components. Colour variation is a problem in histopathology based on transmitted microscopy due to the several factors such as variable chemical colouring/reactivity from different manufacturers/batches of stains, colouring being dependent on staining procedures (timing, concentrations, etc.), and light transmission being a function of section thickness and the use of different scanners.

Because of the lack of efficient tools to share datasets between histology labs, image analysis algorithms are often designed and applied on lab-specific datasets with locally standardized protocols that reduce image variabilities. It is therefore currently unknown if more generic pattern recognition approaches could recognize tissue structures accross several histology labs, which would be of great practical value. Current practice is indeed to train and apply recognition models using specific images for each new lab setting, e.g. [6] recognizes glomerular structures using a lab-specific IHC-stained protocol, [5] compares algorithms to detect mitotic figures from 5 breast cancer H\&E biopsy slides from one hospital,

*e-mail: raphael.maree@ulg.ac.be and [13] recognizes lung tumors in hundreds of experimental mice $H \& E$ whole-slide images but from one research laboratory only. Recently, normalization of H\&E images was proposed [1] to reduce staining variations.

Ideally, pattern recognition algorithms should be able to recognize tissue structures regardless of preparation and imaging conditions, as human experts are capable of doing after histology training. Evaluating automatic algorithms in such realistic settings is challenging. It requires softwares and algorithms able to organize persistently in databases large sets of digital slides from multiple centers, allow semantic annotation of digital slides by experts, as well as protocols and user interfaces to visualize and evaluate algorithm results. In this work we propose to combine and extend softwares (namely Cytomine $^{1}$ [12] and Icy $^{2}$ [2]), and their algorithms (based on machine learning and image processing, respectively) to enable multisite digital pathology studies.

\subsection{Image analysis in renal pathology}

In particular, we will focus here on image analysis in renal pathology. Biopsy is a key diagnostic tool for many renal pathologies because it allows early detection of lesions in the kidney before the clinical symptoms appear. Examination of the biopsy is currently done by several pathologists from a visual examination of biopsy slides stained by several staining, through a microscope. This screening is very tedious and subject to inter and intra-observer variabilities. One of the first visual task consists in counting glomeruli, a critical element of the kidney, in 2D tissue sections to determine if the tissue sampling is representative of the 3D organ. Due to the slicing, the glomeruli on an image section show varying size. Furthermore, the slide images are subject to high variations in staining. As far as we know, there are few works on automatic detection of glomeruli. [9] used an edge detector and genetic algorithms but the validation has been done on 13 glomeruli only. More recently, [6] and [7] proposed a glomeruli detector by using a linear Support Vector Machine (linear-SVM)

\footnotetext{
${ }^{1}$ http://www.cytomine.be/

${ }^{2} \mathrm{http}: / /$ icy.bioimageanalysis.org/
} 
on respectively the Histogram of Oriented Gradients (HOG) and Segmental HOG (S-HOG) features. The HOG reaches the recognition rate of $85 \%$ of glomeruli detection and the S-HOG, improves the result of HOG. However both works are mono-centric studies on mouse renal samples that show less variations than our dataset which comes from a human, multisite study. Indeed, our approach, described in Section 2 , enabled an empirical study on hundreds of tissue sections which results are presented in Section 3.

\section{METHODS}

In this Section, we describe the proposed workflow for effective analysis of large sets of digital slides from multiple centers. Our workflow combines algorithms and protocols to exchange data and results between Icy and Cytomine, two open-source software for bioimage analysis.

\subsection{Image and data storage using Cytomine}

Cytomine is an open-source rich internet application for collaborative analysis of large images [12]. Through HTTP transfers, we centralize images on a Cytomine server and organize them into a project with a user-defined vocabulary of terms describing renal tissue structures. Cytomine supports various slide scanner formats and provide web services to retrieve image thumbnails and tiles corresponding to specific image area at any resolution level. Cytomine data models also allow to store annotation geometries drawn manually or generated by image analysis softwares. Each user or software has its own layer of annotations and these data can be visualized and edited through a web interface and they can also be retrieved or updated by third party softwares through a RESTful API.

\subsection{Icytomine plugin for image and data exchange}

Icy is a collaborative platform that combines a free and opensource Java software designed for bioimage analysis and a community website for contributing and sharing algorithms and protocols. It already implements various image processing algorithms but is does not handle very large images.

Icytomine is a bridge that we developed, schematically represented in Figure 1 to exchange image data and annotations between Icy and Cytomine. Our implementation allows batch processing of hundreds of whole slide images by relying on Icy API and Cytomine RESTful API and JSON lightweight data-interchange format. As explained before, the slide images are first stored in Cytomine database at high magnification. Then Icytomine downloads whole slide image at lower resolution from Cytomine (at 1.5x magnification) to performs section detection using an automatic thresholding method. The detected region of interest (ROI) within

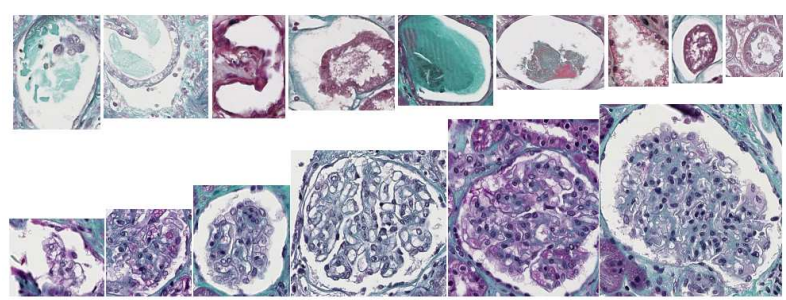

Fig. 2. Example of candidate glomeruli detected by step 1 . Top: False positives, Bottom: True positives.

Icy are converted into corresponding whole slide polygon coordinates in WKT format and sent back to Cytomine using its annotation web services. For glomeruli detection, image tiles of each section are downloaded from Cytomine at higher magnification (20X magnification) and converted to Icy internal data structures for tile processing, as described in the next section.

\subsection{Detection of glomeruli candidates using Icy}

This step (Step 1) aims at detecting glomeruli candidates by using image processing algorithms in Icy. As we want an approach that can detect glomeruli whatever the color staining is, our detection step is based on geometric invariance, more precisely it relies on the ellipse-shape of glomeruli. First, the luminance component is thresholded in order to obtain the lumen regions, from which we fit the ellipses by the Fitzgibbon's method [3]. It minimizes the algebraic distance subject to the constraint $4 a c-b^{2}=1$, by incorporating the ellipticity constraint into the normalization factor. This algorithm requires only five points and has the advantages to be very robust, efficient and simple to implement. However, it detects too many candidates (many of them are non-glomerular structures), as illustrated by Figure 2. We therefore propose to send them back to Cytomine for further processing based on classification algorithms. As these detected objects are stored as ROI objects in Icy, we again used Icytomine to convert them and send their whole-slide coordinates to Cytomine.

\subsection{Automatic Classification of candidate images}

The detection step generates many non-glomerular structures that would require manual intervention to filter them out. In Step 2, we want to evaluate the use of classification algorithms to filter out non-glomerular objects and reduce manual workload. In this work, we propose to evaluate two generic classifiers readily available, namely WND-CHARM [15] and the ET-FL (Extremely Randomized Trees for Feature Learning) tree-based method of [11]. These methods have been shown effective on various classification tasks. However, as our large empirical study reveals that none of these methods 


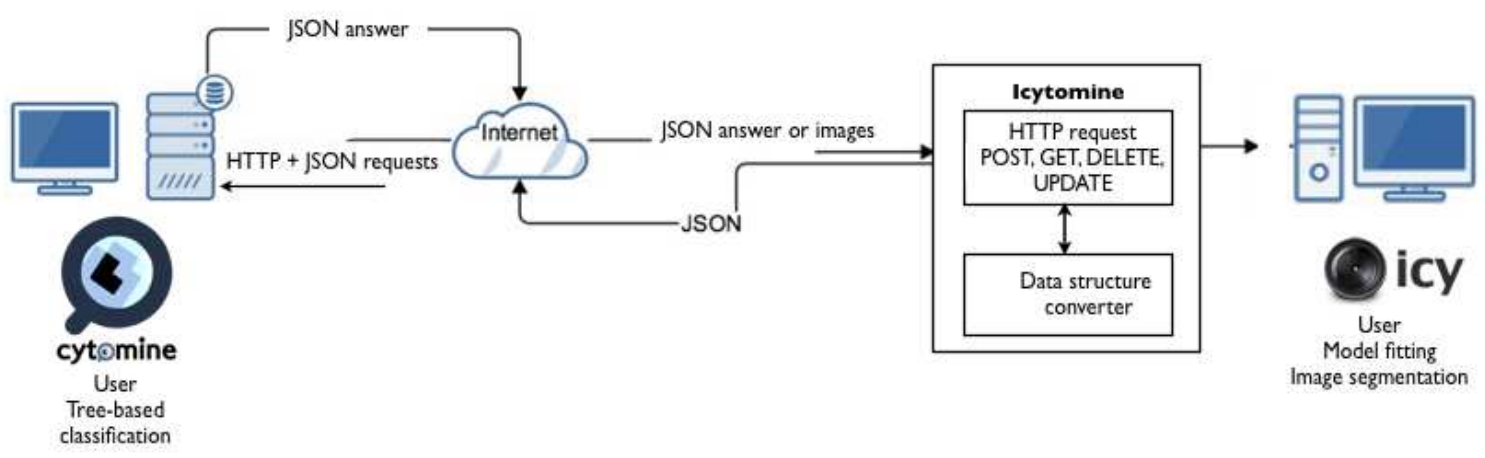

Fig. 1. An overview of our workflow and its communication mechanisms between Icy and Cytomine.

is robust enough on our realistic dataset, we propose to also consider color normalization algorithms to improve recognition performances. More specifically, we combined color normalization with ET-FL method only as this method performed better on non-normalized images.

\subsubsection{Color normalization}

We have tested 4 color normalization methods in order to improve the classification performance: i) the simple color histogram equalization i.e. histogram equalization on each color channel, red, green, blue. ii) Automatic Color Enhancement [4] is an effective color correction and enhancement method based on a simple model of the human visual system and which has a connection with histogram equalization. iii) The color transfer method of Reinhard et al. [16] that maps the images to the color distribution of a target image on a per-pixel basis by equalizing the mean and standard deviations separately for each dimension of a perceptual colorspace. iv) Macenko et al. [10] uses a singular value decomposition (SVD)based approach to directly estimate the color deconvolution matrices.

\subsubsection{Supervised classification}

\subsubsection{WND-CHARM}

We first used [15] that proposed a generic approach for image classification based on the extraction of a large set of features. For each candidate image, it computes polynomial decompositions (Chebyshev-Fourier statistics, Chebyshev statistics, Zernike polynomials), high contrast features (edge and object statistics), pixel and texture statistics (Gabor textures, first four moments, Haralick textures, Multiscale histograms, Tamura textures, Radon). These features are computed on the raw image, transforms of the image (Wavelet, Chebyshev and Fast Fourier transforms), and transforms of transforms of the image (Chebyshev transform of the Fourier transform). In total, this approach generates 2919 features. As in [15], these are then used by a weighted nearest neighbor classifier.

\subsubsection{ET-FL}

We also evaluate systematically the ET-FL classification method of [11] based on random subwindows and extremely randomized tree learning method. This method first performs random extraction of a large number of square subwindows in candidate images then it uses trees to build a novel image description (a global, sparse, feature vector that encodes subwindow frequencies in tree leaves) subsequently classified by a linear SVM classifier. For each of the normalization variant presented previously, we trained models using different configurations of subwindow size intervals (as in [11]), and we report the best obtained result. Default tests were made using 100 training subwindows per candidate image. For each configuration, subwindows are subsequently resized by nearest neighbor interpolation to patches of fixed size $(16 \times 16)$ and their pixel values are encoded in HSV colorspace unless otherwise stated (a few tests were performed using graylevels). For the tree parameters, we used the same set of parameter values: $k=28$ (square root of the total number of attributes), $T=10$, and $n_{\min }=1000$.

\section{EXPERIMENTAL DATA AND RESULTS}

\subsection{Data}

Our dataset contains tissue sections from 200 slides (one slide contains several tissue sections from the same patient) prepared using trichrome Masson protocol within 13 research centers and acquired using a Nanozoomer scanner with $0.452 \mu \mathrm{m}$ pixel resolution. We used a random set of 100 slides (containing a total of 2927 glomeruli and 13648 nonglomerular structures) to train the recognition algorithms, while the remaining sections from 100 slides (containing 


\begin{tabular}{|c|c|c|c|}
\hline Method & Avg. acc. & NonGlom acc. & Glomeruli acc. \\
\hline WND-CHARM GRAY & 73.71 & 81.38 & 47.39 \\
ET-FL GRAY & 87.17 & 96.87 & 53.91 \\
ET-FL HS & 89.01 & 98.85 & 55.29 \\
ET-FL COLOR & 91.67 & 98.61 & 67.89 \\
ET-FL ACE & 91.04 & 98.71 & 64.73 \\
ET-FL RH & 92.62 & 98.95 & 70.93 \\
ET-FL MM & $\mathbf{9 3 . 7 8}$ & $\mathbf{9 8 . 9 7}$ & $\mathbf{7 5 . 9 8}$ \\
\hline
\end{tabular}

Table 1. Summary of recognition performances.

a total of 2853 glomeruli and 9785 non-glomerular structures) were used to evaluated recognition performances. To build the ground truth data of glomeruli and non-glomerular structures in both training and test sets, we used Cytomine web proofreading tools to re-classify manually candidates detected by Step 1 .

\subsection{Results}

Table 1 shows classification accuracies for all method variants on the test set. Our experiments show that using graylevels only yields lower performances for both WND-CHARM and ET-FL classifiers. ET-FL is best combined with the color normalization method of [10]. If we increase the number of training subwindows up to 500, we further increase recognition rate up to 94.74 (98.71 nonglom accuracy, 81.10 glomeruli accuracy) which also corresponds to 0.95 precision, 0.81 recall, and $0.87 F 1$ score. However, although the method succeeds in rejecting most of the non-glomeruli objects, it also incorrectly rejects many glomeruli. Future work should therefore concentrate on balancing true/false positive/negative rates to better meet practical expectations.

\section{CONCLUSION}

In this work we proposed a novel workflow combining Cytomine and Icy softwares and their algorithms for glomeruli detection in human renal biopsies from multiple centers. Although results are not yet satisfactory for practical use, our open-source developments ${ }^{3}$ open the door for large-scale, multisite, digital pathology studies. In the future we will evaluate other color normalization techniques as well as data augmentation and classification approaches inspired by works in natural image classification [8].

\section{ACKNOWLEDGMENTS}

We thank Institut Carnot Maladies Infectieuses for R.M. postdoctoral grant as well as Wallonia (DGO6) for research grant 1017072. We also thank Florian Aubin for preliminary implementation, and Eric Thervet for providing renal slide images.

\footnotetext{
${ }^{3}$ Icytomine plugin will be made available together with the paper publication.
}

\section{REFERENCES}

[1] B.E. Bejnordi, N. Timofeeva, I. Otte-Hller, N. Karssemeijer, and J. van der Laak. Quantitative analysis of stain variability in histology slides and an algorithm for standardization. In SPIE, editor, Proc. Medical Imaging: Digital Pathology, volume 9041, March 2014.

[2] F. de Chaumont, S. Dallongeville, N. Chenouard, N. Herve, S. Pop, T. Provoost, V. Meas-Yedid, P. Pankajakshan, T. Lecomte, Y. Le Montagner, T. Lagache, A. Dufour, and J. C. Olivo-Marin. Icy: an open bioimage informatics platform for extended reproducible research. Nat Methods, 9(7):690-6, 2012.

[3] A. W. Fitzgibbon, M. Pilu, and R. B. Fisher. Direct least-squares fitting of ellipses. IEEE Transactions on PAMI, 21(5):476-480, May 1999.

[4] Pascal Getreuer. Automatic Color Enhancement (ACE) and its Fast Implementation. Image Processing On Line, 2:266-277, 2012.

[5] Alessandro Giusti, Dan Claudiu Ciresan, Claudio Caccia, Jürgen Schmidhuber, and Luca Maria Gambardella. A comparison of algorithms and humans for mitosis detection. In International Symposium on Biomedical Imaging (ISBI), 2014.

[6] Y. Hirohashi, R. Relator, T. Kakimoto, R. Saito, Y. Horai, A. Fukunari, H. Utsumi, K. Okada, and T. Kato. Automated quantitative image analysis of glomerular desmin immunostaining as a sensitive injury marker in spontaneously diabetic torii rats. Journal of Biomedical Image Processing, 1(1):20-28, May 2014.

[7] T. Kato, R. Relator, H. Ngouv, Y. Hirohashi, O. Takaki, T. Kakimoto, and K. Okada. Segmental hog: new descriptor for glomerulus detection in kidney microscopy image. BMC Bioinformatics, 16:316, 2015.

[8] Alex Krizhevsky, Ilya Sutskever, and Geoffrey E. Hinton. Imagenet classification with deep convolutional neural networks. In F. Pereira, C.J.C. Burges, L. Bottou, and K.Q. Weinberger, editors, Advances in Neural Information Processing Systems 25, pages 1097-1105. Curran Associates, Inc., 2012.

[9] J. Ma and Zhang J. an Hu J. Glomerulus extraction by using genetic algorithm for edge patching. In Proc. of IEEE Congress on Evolutionary Computation, pages 2474-2479, 2009.

[10] M. Macenko, M. Niethammer, J.S. Marron, D. Borland, J.T. Woosley, Xiaojun Guan, C. Schmitt, and N.E. Thomas. A method for normalizing histology slides for quantitative analysis. In Proc. ISBI, pages 1107-1110, June 2009.

[11] R. Marée, P. Geurts, and L. Wehenkel. Towards generic image classification: a extensive empirical study. Technical report, University of Lige, December 2014. Available online at http://orbi.ulg.ac.be/handle/2268/175525.

[12] R. Marée, L. Rollus, B. Stevens, R. Hoyoux, G. Louppe, R. Vandaele, J.M. Begon, P. Kainz, P. Geurts, and L. Wehenkel. Collaborative analysis of multi-gigapixel imaging data using cytomine. Accepted for publication (minor revision) in Bioinformatics, 2015. http://www.cytomine.be/.

[13] R. Marée, L. Rollus, B. Stevens, G. Louppe, O. Caubo, N. Rocks, S. Bekaert, D. Cataldo, and L. Wehenkel. A hybrid human-computer approach for large-scale image-based measurements using web services and machine learning. Proc. ISBI, pages 902-906, 2014.

[14] MT. McCann, C. Castro, JA. Ozolek, B. Parvin, and J. Kovacevic. Automated histology analysis: opportunities for signal processing. IEEE Signal Processing, 32(1):78-87, 2014.

[15] N. Orlov, L. Shamir, T. Macura, J. Johnston, D. M. Eckley, and I. Goldberg. Wnd-charm: Multi-purpose image classification using compound transforms. Pattern Recognition Letters, 29(11):1684-1693, 2008.

[16] E. Reinhard, M. Adhikhmin, B. Gooch, and P. Shirley. Color transfer between images. Computer Graphics and Applications, IEEE, 21(5):34-41, Sep 2001. 